\title{
Digestion
}

Abe, Y. 40

Ando, T. 47

Asaishi, K. 59

Asaka, M. 34

Ashida, K. 23

Asonuma, S. 40

Atsumi, T. 40

Chayama, K. 53

Domon, K. 29

Eguchi, T. 23

Fujimura, T. 6

Fukuchi, T. 23

Furuhata, T. 29

Furuichi, Y. 17

Fushida, S. 6

Goto, M. 59

Harmon, J.W. 6

Hata, T. 34

Hattori, T. 6

Hayashi, N. 53

Higuchi, K. 59

Hirota, J. 34
Hoshi, T. 40

Hosokawa, A. 47

Hoteya, S. 29

Ichimura, S. 17

Iijima, K. 40

Iizuka, T. 29

Imai, A. 34

Imai, Y. 17

Ito, D. 23

Ito, H. 40

Kajiura, S. 47

Kamada, G. 34

Kanao, H. 53

Kanno, T. 40

Kasie, M. 29

Kato, M. 34

Kawabe, S. 59

Kawaguchi, S. 23

Kawai, T. 17

Kii, T. 59

Kikuchi, D. 29

Koga, H. 23

Koike, T. 40

Konishi, H. 40

Kudo, T. 34

Kuwakado, S. 59
Matsui, A. 29

Mitani, T. 29

Miwa, K. 6

Miyamoto, T. 59

Miyashita, T. 6

Miyata, Y. 17

Moriyasu, F. 17

Murashima, E. 17

Nagatani, Y. 23

Nakagawa, M. 34

Nakagawa, S. 34

Nakamura, I. 17

Nakamura, M. 29

Nakata, K. 47

Ninomiya, I. 6

Nishida, M. 34

Nishida, U. 34

Nishijima, K. 6

Nishitani, H. 59

Ogawa, O. 29

Ohashi, R. 23

Ohta, T. 6

Ohyauchi, M. 40

Oka, S. 53

Ono, S. 34

Oyama, K. 6
Saito, S. 47

Sano, N. 40

Sano, T. 17

Sasaki, S. 6

Senda, K. 23

Shah, F.A. 6

Shimada, N. 40

Shimizu, Y. 34

Shimosegawa, T. 40

Shinomura, Y. 5

Sugimoto, K. 17

Sugiyama, T. 47

Taira, J. 17

Takeda, H. 34

Tanaka, S. 53

Tanaka, T. 23

Tsujii, M. 12

Ubukata, S. 23

Ueda, A. 23

Yamada, A. 29

Yamashita, H. 23

Yamashita, S. 29

Yoshida, M. 59

Yoshida, S. 53

Yoshida, T. 34

\section{Subject Index Vol. 87, No. 1, 2013}

Argon plasma coagulation 17

Barrett's adenocarcinoma 29

- esophagus 6

Bevacizumab 59

Cancer stem cells 12

Carcinogenesis 6

Chemoprevention 6

Colorectal cancer 12, 59

- tumor 53

COX-2 12

Drink test 34

Endoscopic hemostasis 40

- submucosal dissection 29
Esophageal adenocarcinoma 6

- cancer 6

Esophagogastric junctional cancer 29

First-line chemotherapy 59

Fluoropyrimidine 59

Frail patients 59

Functional gastroduodenal disorders 34

Gastric accommodation 34

- emptying 34

Gastrointestinal stromal tumors 47

Great earthquake 40
Hemorrhagic ulcer 40

High-resolution manometry 23

Histogenesis 6

Hypermethylation 12

Hypomethylation 12

Imatinib mesylate 47

Liver cirrhosis 17

Lymphovascular invasion 29

Magnification 53

Malignancy 47

Narrow-band imaging 53

New classification of achalasia 23
Pit pattern 53

Pneumatic dilatation 23

Primary achalasia 23

S-1 59

Sedation 17

Stemness 12

Subepithelial invasion 29

Submucosal invasion 29

Transnasal endoscope 17

Ultrasonography 34

Varices 17

Visceral hypersensitivity 34 